\title{
Psychological Pricing in Mergers \& Acquisitions using Game Theory
}

\author{
$\underline{\text { N. Agarwal }}^{\text {a }}$ and P. Zeephongsekul ${ }^{\text {a }}$ \\ ${ }^{a}$ School of Mathematics and Geospatial Sciences, RMIT University, Melbourne \\ Email:nipun1@msn.com
}

\begin{abstract}
Mergers and acquisitions pricing is usually a difficult task as it is hard to estimate the price to be paid to purchase the target company. Corporate Finance valuations provide some support, however, Baker, Pan and Wurgler (2009) explain that psychological pricing factors exist and the final price can be quite different from the initial valuations. This paper develops a two-person Merger \& Acquisition model that intends to provide a simulation model in order to understand if the final negotiated price of an Merger \& Acquisition transaction equals the mid-point of the acquirer's offer and target's bid price. Also, it reviews behavioural factors that can be part of Merger \& Acquisition valuation and uses the model to simulate results to identify, in this case, if it is better for the acquirer to be risk taking or risk averse and for the target to be optimistic or pessimistic.
\end{abstract}

Results show that the equilibrium price (Nash Equilibrium Point) is closer to the acquirer's offer price. However, through the simulation of the behavioural factors it is seen that the most optimal outcome for the acquirer is to be a risk taker and for the target to be pessimistic. This seems like a suitable option because the acquirer will be willing to provide a higher offer and the target will be more open to accept a reasonable offer. Any of the other three options are less likely to provide such a suitable outcome.

This paper has only undertaken preliminary research in this area, however it shows that there is a possibility to simulate behavioural factors in valuing two-person Merger \& Acquisition transactions. This is specially the case as Merger \& Acquisition valuations include a significant factor of psychological pricing that cannot be explained by traditional Corporate Finance models. Hopefully, this research will provide a platform for other models to be developed to simulate behavioural factors related to Merger \& Acquisition valuations.

Keywords: Mergers and acquisitions, Game theory, Prospect theory. 


\section{INTRODUCTION}

Mergers and Acquisitions (M\&A) is a significant research area in the field of Corporate Finance. There is substantial finance literature on how M\&A transactions can be valued. At present, the investment banking industry uses the concepts of balance sheet valuation models (book value, liquidity value and replacement cost), dividend discount model, price earnings ratio, discount cash flow analysis, transaction multiples and economic profit model to price such transactions (see Marren 1993 for more information). M\&A pricing has been a challenging task, primarily as different valuation methods can provide different results.

Due to a biased system of beliefs and psychological anchoring in M\&A negotiation these models fall short in providing suitable estimates of offer prices that will be acceptable for M\&A transactions and usually under or over-value such transactions. Baker, Pan and Wurgler (2009) and Baker, Ruback and Wurgler (2004) indicate that behavioural factors can impact the pricing of targets in M\&A transactions.

M\&A pricing can been seen as a negotiation between the acquirer and the target to agree on a price at which the acquirer can purchase the target. As a result, we need to use game theory to review such negotiations. Previous research shows that game theory provides an insight into strategic human interaction in such financial negotiations. Contemporary game theory has two forms: non co-operative game theory (Nash 1951) and co-operative game theory (Von Neumann and Morgernstern 1944; Shapley 1953; Shapley 1977; Shapley and Shubik 1954; Luce and Raiffa 1957; Aumann and Drèze 1974; Myerson 1977). Von Neumann and Morgenstern (1944) and Nash $(1950 ; 1951)$ have suggested two game theory approaches to resolve bargaining problems: axiomatic or strategic. The axiomatic approach (sometimes called co-operative theory) assists by providing a set of valuable axioms. On the other hand, the strategic approach models outcomes in a non co-operative game. Mergers and acquisitions would come under the premise of zero sum or non cooperative games.

The objective of this paper is to develop a two-person M\&A game theoretic model that will analyse if the offer price provided by an acquirer should be equal to the mid-point of the acquirer's offer and the target's bid price? It will also analyse if the acquirer will do better by being risk taking or risk averse and if the target will do better by optimistic or pessimistic in this model. The next section of this paper will explain this model. While, the third section will discuss the results of a simulation that explains what behaviour an acquirer (risk taking or risk averse) and target (optimistic or pessimistic) should show in such a negotiation. Finally, the last section of this paper will summarise the discussion.

\section{TWO-PERSON MERGER \& ACQUISITION MODEL}

The two-person M\&A model is an incomplete information game between the acquirer and the target, where both players need to agree to a price that will be suitable to them for the sale of the target company to the acquirer. The extensive form for this game can be seen in figure 1 that depicts a three stage game between the acquirer (buyer) and target (seller). In this game, the acquirer can choose either the "Increase Bid" or "Reduce Bid" strategy and the target can choose the "Increase Bid" or "Stable Bid" strategy. This game also has two chance nodes which are associated with probabilities related to the expected behaviour of the acquirer if it is either "risk taking" or "risk averse" and if the target is "optimistic" or "pessimistic". Here, probabilities are assigned based on the expected behaviour (type) that the acquirer or target may show. If these probabilities are changed then the equilibrium point in this game may change as well. Before we move further, we should note that the pay-offs for each strategy is relative to any other strategy in this game. So, the "Increase Bid" strategy has a pay-off of -1 (for the acquirer as it needs to pay more to purchase the target) and +1 (for the target if we consider a zero-sum game). However, we use the concept called Prospect Theory (Khaneman and Tversky 1979), which states that humans prefer positive events at least twice as much as negative events. Therefore, positive events are divided by 2.25 (loss aversion co-efficient, see Khaneman and Tversky 1992) to equate them to negative events (see Metzger and Reiger 2010 for application of prospect theory to game theory). As a result all positive events would have a pay-off equal to +0.44 instead of +1 . On the other hand, the "Reduce Bid" strategy has a pay-off of +1 (for the acquirer or +0.44 after considering prospect theory) and -1 (for the target). The "Stable Bid" strategy will be associated with a pay-off equal to 0 .

The extensive form (diagrammatic form of the game providing the sequences of strategies played by each player) and normal form (tabular form of the game providing the final outcomes of each strategy pair assuming strategies are played simultaneously) of the two-person M\&A model are provided in figure 1 and table 1 respectively. The extensive and normal form have been developed using the Gambit (version 0.2007.12.04) software package (http://www.gambit-project.org/doc/index.html). 


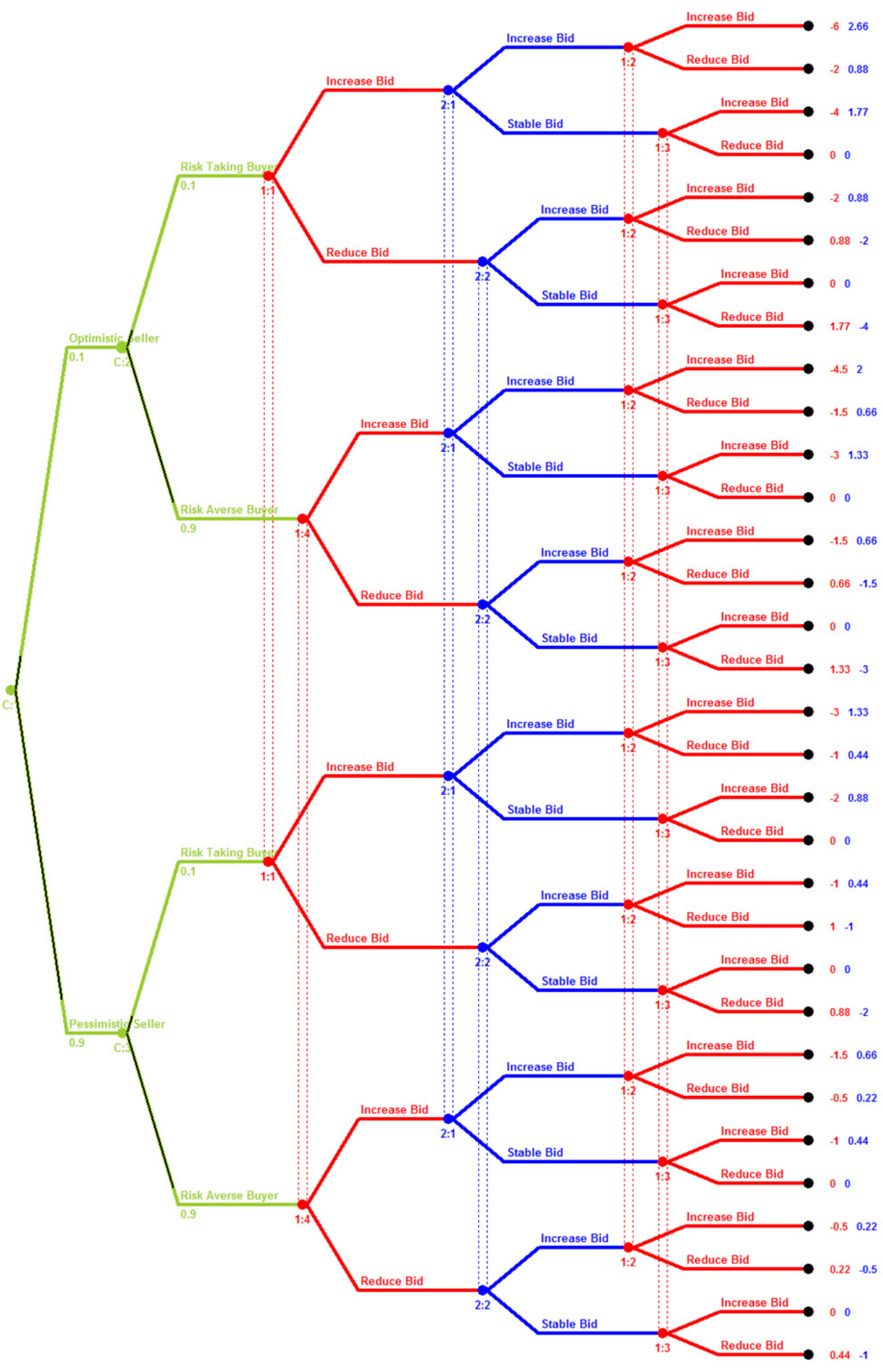

Figure 1. Extensive form of the Two-person Merger \& Acquisition game model 
In the extensive form of the two-person M\&A model (figure 1), the base case is when the acquirer is risk taking and the target is pessimistic. Further, the pay-offs are decreased by 50 per cent from the base case, if the acquirer is risk averse and the target is pessimistic. On the contrary, the pay-offs are increased by 50 per cent, if the acquirer is risk averse, but the target is optimistic. The pay-offs are increased 100 per cent from the base case when the acquirer is risk taking and the target is optimistic. The intent of this concept is that a risk taking acquirer will be willing to pay more compared to a risk averse acquirer. While, an optimistic target will ask for more in order to sell his company compared to a pessimistic target.

It is important to recall that we are dealing with an incomplete game, where the acquirer does not know if the target is optimistic or pessimistic and the target does not know if the acquirer is risk taking or risk averse. Due to this fact, we have information sets around similar node types. For example, if we need to calculate the pay-off for the acquirer when he uses the "IIII" strategy against the "II" strategy used by the target. We will need to add the pay-offs for each instance where the acquirer will use the "IIII" strategy multiplied by the acquirer and target's probabilities. So, this would be $(1.0) \times(0.1) \times(-6)+(1.0) \times$ $(0.9) \times(-4.5)+(0.0) \times(0.1) \times(-3)+(0.0) \times$ $(0.9) \times(-1.5)$, which would equal -4.65 . Similarly, the pay-off for the target will be calculated being +2.07 .

Nash (1951) states that players who play strategies that coincide with the Nash Equilibrium Point (NEP) will succeed against any opponent who differs from their strategy that coincides with an NEP. Therefore, both the Acquirer and Target can select from a set of strategies $S$, where a combination of strategies $S^{*}=\left(s_{1}^{*}, s_{2}^{*}\right) \in S$ is an NEP if it satisfies the property:

$$
\begin{array}{ll}
u_{1}\left(s_{1}^{*}, s_{2}^{*}\right) \geq u_{1}\left(s_{1}, s_{2}^{*}\right) & \forall s_{1} \in S_{1} \\
u_{2}\left(s_{1}^{*}, s_{2}^{*}\right) \geq u_{2}\left(s_{1}^{*}, s_{2}\right) & \forall s_{2} \in S_{2}
\end{array}
$$

Where, $u_{1}$ is the utility for Player 1 and $u_{2}$ is the utility for Player 2 for playing their strategy.

The Nash Equilibrium Point (NEP) for this game will be the strategy "RRRR" or "RRIR" played by the acquirer and the strategy "II" played by the target, which will result in a pay-off of $(0.68,-1.55)$. Neither that acquirer nor the target will choose any other strategy, otherwise they run the risk of ending up with a lower pay-off. This shows that the NEP (equilibrium state) for this game is closer to the acquirer's offer than the target's bid price, where the acquirer is risk averse and the target is optimistic. It would be interesting to analyse the behaviour of the acquirer and target further to understand what behaviour will maximize the payoff for both the players.

\section{OPTIMAL BEHAVIOR OF THE ACQUIRER AND TARGET}

If we change the probabilities for the acquirer's risk taking - risk averse behaviour and the target's optimistic - pessimistic behaviour in the two-person M\&A model. Then, we could possibly understand what behaviour would be best for these players to adopt in such a game? Four possible combinations can occur in this model, which are:

\footnotetext{
1. Target: Optimistic; Acquirer: Risk Taking

2. Target: Pessimistic; Acquirer: Risk Taking

3. Target: Optimistic; Acquirer: Risk Averse

4. Target: Pessimistic; Acquirer: Risk Averse
} 
In the real world, M\&A transactions are conducted in similar circumstances as depicted by the two-person M\&A model - as both incomplete information and prospect theory preferences exist. Due to these factors, valuations can be higher or lower than what Corporate Finance valuation models would recommend - some relevant examples are the Centro Properties Fund (US assets) that has been recently bought by the Blackstone group (Centro's US assets were sold at a larger discount due to indebtedness), MySpace was bought by News Corporation (for a premium at \$580 million, but now News Corp wants to get rid of it for a substantially lower price) and Bank of America purchased Merrill Lynch at a substantial discount. Other more recent examples are the merger negotiation between SAB Miller and Foster's group, AMP and AXA Asia Pacific and others have psychological pricing variances.

The two-person M\&A model has identified the behaviours (types) of the acquirer (risk taking - risk averse) and target (optimistic - pessimistic) that will change between players depending on the M\&A transaction. It is also often hard to find the behaviours of opponents and the associate probabilities to these behaviours in order to calculate potential NEPs for such games. However, this model does provide us some significant insight on how such M\&A transactions can be analysed more accurately, which will help the acquirer or target, develop and simulate strategies that it can follow against its opponent. An example of such a simulation of the behavioural probabilities of the acquirer and target is provided in Table 2 .

A simulation of the two-person M\&A model will help us understand if the acquirer should choose to be risk taking or risk averse and the target will be able to gauge if he should act optimistically or pessimistically. The data from the simulation of the two-person M\&A model in table 2 has shown that the Nash Equilibrium Point for such a series of games lies at $(2,-2)$, which relates to the acquirer being a risk taker and the target acting pessimistically. In an everyday scenario such a result makes sense because if the acquirer is a risk taker, he will be ready to offer more for the sale. While, on the other hand if the target is pessimistic then he will be more likely to negotiate with the acquirer on a reasonable price.

However, if we consider the opposite situation, where the acquirer is risk averse and the target is optimistic, then it is less likely that a successful negotiation will result. As, the acquirer will be unwilling to negotiate a higher price for the merger and the target will be unlikely to accept a lower price for this transaction. Such a situation will most likely result in the merger to break down. The situation will not be much different when the acquirer is risk averse and the target is pessimistic - in this case, the M\&A valuation will likely favour the acquirer compared to the target, which may result in the target discontinuing the transaction. However, if the acquirer was risk taking and the target was optimistic, there still is a risk that the target may ask for more than the acquirer is willing to offer, which may result in the merger talks being stalled.

In everyday business practice, we find all different varieties of people, some who are risk taking, while others are risk averse. Also, some are optimistic and others are pessimistic. It is possible that not only these but other behavioural traits can be used and even combined to develop more realistic M\&A valuation games. The pay-offs will change based on the behavioural characteristics and strategies that are chosen to represent the M\&A valuation game. It is inevitable that game theory will be used more often for M\&A valuation as it helps support the psychological pricing variances that are not available through traditional Corporate Finance valuation techniques. Those techniques do provide some theoretical basis to the M\&A valuation decision, however due to behavioural biases, it is not possible to obtain an understanding of what would be considered as the most optimal offer (Nash Equilibrium Point) that will provide the best result to the acquirer and the target. Further, the concept of prospect theory that has such a significant impact on pricing, cannot be considered by the traditional valuation model. Therefore, the two-person M\&A model has been developed to help provide a tool that could be used to simulate M\&A pricing that can provide a platform for valuing potential M\&A transaction that relate to real world situations. 


\section{DISCUSSION AND CONCLUSIONS}

Results of the two-person M\&A model show that the strategies used by the acquirer are "RRRR" or "RRIR" against the target's strategies "II". As a result, the pay-off to the acquirer is +0.68 and the target is -1.55 . When this model is used to simulate the behaviour of the acquirer and target, it is seen that it would be better for the acquirer to be risk taking and the target to be pessimistic. This would help as the acquirer would be willing to pay more, while the target will be willing to accept less for the sale of the target company.

It is to be noted that these are preliminary results from this research, as this model is developed further the results and discussion will possibly help provide greater insight into psychological pricing of M\&A transactions.

\section{REFERENCES}

Aumann, R. J. and Drèze, J. (1974). Cooperative Games with Coalition Structures. International Journal of Game Theory, 3, 217-237.

Baker, M., Ruback, R.S. and Wurgler, J. (2004). Behavioral corporate finance: A survey. NBER working paper, 10863, Cambridge, Massachusetts.

Baker, M., Pan, X. and Wurgler, J. (2009). The Psychological Pricing in Mergers and Acquisitions. NBER working paper, Cambridge, Massachusetts.

Luce, R.D. and Raiffa, H. (1957). Game and Decisions: Introduction and critical survey. John Wiley, New York.

Khaneman D. and Tversky, A. (1979). Prospect Theory: An Analysis of Decision under Risk, Econometrica, 47(2), 263-292.

Khaneman D. and Tversky, A. (1992). Advances in prospect theory: Cumulative representation of uncertainty. Journal of Risk and Uncertainty, 5(4), 297-323.

Marren, J.H. (1993). Mergers \& Acquisitions: A valuation handbook. Business One Irwin, Homewood, Illinois.

Metzger, L.P. and Reiger M.O. (2010). Equilibria in games with prospect theory preferences. Working paper no. 598, National Centre of Competence in Research Financial Valuation and Risk Management, Zurich 2009.

Myerson, R. B. (1977). Graphs and Cooperation in Games. Mathematics of Operations Research, 2, 225229.

Nash, J.F. (1950). The Bargaining Problem. Econometrica, 18, 155-162.

Nash J.F. (1951). Non-cooperative games. Annals of Mathematics, 54, 286-295.

Shapley, L. S. (1953). A Value for n-Person Games. In H. W. Kuhn and A. W. Tucker (eds.). Contributions to the Theory of Games II (Annals of Mathematics Studies 28), Princeton University Press, 307-317.

Shapley, L. S. (1977). A Comparison of Power Indices and a Nonsymmetric Generalization. P-5872. Rand Corporation, Santa Monica.

Shapley, L. S. and Shubik, M. (1954). A Method for Evaluating the Distribution of Power in a Committee System. American Political Science Review, 48, 787-792.

Von Neumann, J. and O. Morgenstern (1944). Theory of Games and Economic Behavior, Princeton University Press, New Jersey. 